\title{
Actualism and Modal Semantics
}

\author{
José L. Zalabardo ${ }^{1}$ iD
}

Received: 14 March 2017 / Accepted: 2 October 2017 /Published online: 22 November 2017

(C) The Author(s) 2017. This article is an open access publication

\begin{abstract}
According to actualism, modal reality is constructed out of valuations (combinations of truth values for all propositions). According to possibilism, modal reality consists in a set of possible worlds, conceived as independent objects that assign truth values to propositions. According to possibilism, accounts of modal reality can intelligibly disagree with each other even if they agree on which valuations are contained in modal reality. According to actualism, these disagreements (possibilist disagreements) are completely unintelligible. An essentially actualist semantics for modal propositional logic specifies which sets of valuations are compatible with the meanings of the truth-functional connectives and modal operators without drawing on formal resources that would enable us to represent possibilist disagreements. The paper discusses the availability of an essentially actualist semantics for modal propositional logic. I argue that the standard Kripkean semantics is not essentially actualist and that other extant approaches also fail to provide a satisfactory essentially actualist semantics. I end by describing an essentialist actualist semantics for modal propositional logic.
\end{abstract}

\section{Two Conceptions of Modal Reality}

In his paper, "Theories of Actuality", Robert Adams provides a characterization of the debate between actualism and possibilism regarding possible worlds. Possibilism, on Adams's characterization,

is the view that there are nonactual possible worlds and that the notion of a possible world is not to be analyzed in terms of actual things. (Adams 1974: 224)

Actualism, on the other hand,

José L. Zalabardo

j.zalabardo@ucl.ac.uk

1 University College London, Gower Street, London WC1E 6BT, UK 
is the view that if there are any true statements in which there are said to be nonactual possible worlds, they must be reducible to statements in which the only things there are said to be are things which there are in the actual world and which are not identical with nonactual possibles. The actualist will not agree that there are nonactual possible worlds, if the notion of possible worlds is to be regarded as primitive. (Adams 1974: 224)

Adams puts forward a strategy for construing talk of possible worlds within an actualist perspective. He refers to it as the true-story theory:

The true-story theorist $[. .$.$] regards a merely possible world as logically construct-$ ed out of the set of propositions that are true in it, and he sees the truth of a proposition in a possible world as basically a matter of relations of consistency among propositions, rather than of correspondence with an independent object. (Adams 1974: 227) ${ }^{1}$

Propositions, if they exist, exist in actuality. ${ }^{2}$ Hence, if possible worlds could be constructed along the lines of the true-story theory, actualism, on Adams's characterization, would be vindicated.

We can use these ideas to formulate a contrast between two conceptions of the fundamental character of modal reality - of the aspects of reality to which our modal talk is answerable. The actualist will treat modal reality as a logical construction out of sets of propositions, or, equivalently, out of valuations (functions from the set of propositions to the values True and False). On the version of actualism that I want to concentrate on, modal reality is logically constructed out of a set of total valuations, with all propositions, modal as well as non-modal, in their domain. ${ }^{3}$ For the actualist, once we have specified which total valuations it contains, we will have given a complete characterization of modal reality. ${ }^{4}$

For possibilism, by contrast, modal reality consists of a set of possible worlds, conceived as independent objects, all but one of which do not exist in actuality. Each possible world bestows a truth value on each proposition, according to whether or not things are at that world as the proposition represents them as being. In this way, modal reality, for the possibilist, singles out a set of valuations. But for the possibilist, the set of valuations it singles out is not all there is to modal reality. Possible worlds have properties and bear relations to one another that might not be reflected in the truth values they bestow on propositions.

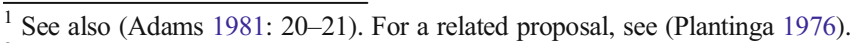

${ }^{2}$ I am not taking account of the thought, explored by Adams in a later paper, that if certain non-actual worlds were actual there would be true propositions that do not actually exist. See (Adams 1981: 21-22).

${ }^{3}$ It's not clear whether the logical construction of possible worlds advocated in (Adams 1974) is based on total valuations or non-modal valuations (whether the sets of propositions in terms of which they are constructed include modal propositions). However, (Adams 1981: 31) presents the view that modal propositions are not to be included in world-stories as an alternative to the position advanced in the paper. This would suggest that Adams's construction is based on total valuations.

${ }^{4}$ Actualism does not carry a commitment to a specific view as to which total valuations are contained in modal reality or to any substantive conditions that the set of total valuations contained in modal reality would have to satisfy. Actualism is simply the view that there is no more to modal reality than the set of total valuations it contains.
} 
Which of these positions we adopt will have consequences for which differences between accounts of modal reality we are prepared to treat as intelligible. The crucial point here is whether accounts of modal reality can disagree with one another while agreeing on which valuations it contains. For the possibilist, on the one hand, this kind of disagreement is perfectly intelligible. Accounts of modal reality can agree on which valuations are generated by possible worlds while disagreeing on properties of and relations between possible worlds that are not reflected in the truth values that propositions receive from them. I am going to refer to disagreements of this kind between accounts of modal reality as possibilist disagreements. Possibilism is committed to the intelligibility of possibilist disagreements. For the actualist, on the other hand, possibilist disagreements are completely unintelligible. Once we have agreed on which valuations modal reality contains, for the actualist, there is nothing left for us to agree or disagree about concerning modal reality. ${ }^{5}$

\section{Semantics for Possibilists and Actualists}

A coherent account of modal reality needs to be compatible with the meanings of logical operators, and both possibilists and actualists face the task of specifying which accounts satisfy this criterion. This is the job of a semantic theory. Here, I am going to concentrate on the sentential aspect of the task, on the compatibility of accounts of modal reality with the meanings of the truth-functional connectives and modal operators, with the modal operators taken to express metaphysical modality. Possibilists and actualists alike will need to provide a semantics for modal propositional logic, but they will take different approaches to the task.

For the possibilist, possible worlds bestow truth values on propositions. They will do this in ways that are compatible with the meanings of the logical operators, and a coherent account of modal reality will have to abide by this restriction. For example, on a coherent account of modal reality, a possible world cannot give the same value to a proposition and its negation, or the value True to a conjunction and the value False to one of its conjuncts. Likewise, in a coherent account of modal reality, a possible world cannot give the value False to necessarily-p if every world gives the value True to $p$, nor can a possible world give the value True to possibly-p if every world gives the value False to p. The possibilist's goal, then, is to specify how a set of possible worlds can assign to proposition values that are compatible with the meanings of the logical operators.

The actualist will approach the task in a different way. For her, a coherent account of modal reality is a set of valuations that are compatible with the meanings of the logical operators. Her goal is to specify which sets of valuations satisfy this criterion. Thus, for example, she will need to exclude sets containing valuations that assign the same truth value to a proposition and its negation, or valuations that assign the value True to a conjunction and the value False to one of its conjuncts, as well as any set containing valuations that assign the value False to necessarily-p if all the valuations in the set

\footnotetext{
${ }^{5}$ As illustrations of a possibilist disagreement (details below) consider two accounts of modal reality which agree on which valuations are generated by possible worlds but disagree about which worlds generate these valuations, or about how many possible worlds generate a given valuation.
} 
assign the value True to $\mathrm{p}$ and sets containing valuations that assign the value True to possibly-p if all the valuations in the set assign the value False to p. The actualist's goal is to specify which sets of valuations are compatible with the meanings of the logical operators.

These two approaches to the task are clearly related, and it might seem that the differences between them are merely verbal - that the possibilist's talk of possible worlds bestowing truth values on propositions adds nothing of substance to the actualist's talk of valuations being contained in modal reality. However a difference arises from the possibilist's need to make room for possibilist disagreements. The possibilist semantic framework will have to have the resources to accommodate differences between coherent accounts of modal reality that agree with one another on which valuations are generated by possible worlds. The actualist, by contrast, will not be subject to this constraint.

It follows from this that the possibilist faces a strictly harder task than the actualist does. On the one hand, every solution to the possibilist task can be adapted by the actualist for her purposes. By specifying how propositions can receive from a set of possible worlds truth values that are compatible with the meanings of the logical operators, a possibilist semantics will be specifying, indirectly, which sets of valuations are compatible with the meanings of the logical operators. In this way, every possibilist semantics can be converted into an actualist semantics. On the other hand, a solution to the actualist task might not be usable by the possibilist. We might in principle be able to specify the sets of valuations that are compatible with the meanings of the logical operators without drawing on formal resources that would enable us to make room for possibilist disagreements. ${ }^{6}$ I am going to refer to a semantics for modal propositional logic that satisfies this description as an essentially actualist semantics. An essentially actualist semantics will solve the actualist task but not the possibilist task, since it will not have the resources to make room for possibilist disagreements.

My goal in the remainder of this paper is to discuss the availability of an essentially actualist semantics for modal propositional logic. I am going to argue first that the standard Kripkean semantics for modal propositional logic is not essentially actualist, as it has the resources for making sense of possibilist disagreements, and I will present specific ways in which Kripkean semantics falls short of essential actualism. I will then argue that other extant alternatives also fail to provide what a sensible actualist would need. I will end by describing a semantics for modal propositional logic that does the job - it specifies which sets of valuations are compatible with the meanings of the logical operators without invoking formal tools that would enable us to make sense of possibilist disagreements.

\section{Kripkean Semantics}

Let us focus on a language, MPL, for modal propositional logic with $\neg, \wedge, \square$ and $\diamond$, as its only logical operators, representing, as usual, negation, conjunction, necessity, and possibility. A set of MPL-valuations may or may not be compatible with the meanings

\footnotetext{
${ }^{6}$ As we shall see below, the formal resources that an essentially actualist semantics cannot invoke include the possible worlds and accessibility relation of standard Kripkean modal semantics.
} 
that we want to ascribe to our logical operators. A set will fail the test if, for example, it contains a valuation that assigns the same value to $\phi$ and $\neg \phi$, for any sentence $\phi$, or the value True to $\phi \wedge \psi$ and the value False to $\phi$. Likewise, a set will fail the test if it contains a valuation assigning the value True to $\diamond \phi$ but no valuation assigning the value True to $\phi$, or if it contains a valuation assigning the value False to $\square \phi$ but no valuation assigning the value False to $\phi$. Let say that a set of MPL-valuations is admissible just in case it is compatible with the meanings of the logical operators. Specifying which sets of MPL-valuations are admissible is the goal of a semantics for MPL.

The standard approach to this task deploys the tools introduced by Kripke (1963). Kripke's semantic framework for modal propositional logic is based on what I am going to call K-models. A K-model $M$ is an ordered triple $<W_{M}, R_{M}, S_{M}>$, where $W_{M}$ is a (non-empty) set of objects (the possible worlds), $\mathrm{R}_{\mathrm{M}}$ is a binary relation on $\mathrm{W}_{\mathrm{M}}$ (the accessibility relation), and $\mathrm{S}_{\mathrm{M}}$ is a function pairing each element of $\mathrm{W}_{\mathrm{M}}$ and atomic sentence with a unique truth value - the value that the atomic sentence has at that world. ${ }^{7}$ For every K-model M, we now define a function $\mathrm{V}_{\mathrm{M}}$, the canonical extension of $\mathrm{S}_{M}$ (with respect to $M$ ), pairing each MPL-sentence and element of $\mathrm{W}_{M}$ with a unique truth value, the value that the sentence has at that world. $V_{M}$ is defined by recursion. For the base, we have that for every atomic sentence $\alpha$, and every $\mathrm{w} \in \mathrm{W}_{\mathrm{M}}$, $\mathrm{V}_{\mathrm{M}}(\alpha, \mathrm{w})=\mathrm{S}_{\mathrm{M}}(\alpha, \mathrm{w})$. The recursive clauses go as follows, for all MPL-sentences $\phi$, $\psi$, and every $\mathrm{w} \in \mathrm{W}_{\mathrm{M}}$ :

$$
\begin{aligned}
& (\neg) \mathrm{V}_{\mathrm{M}}(\neg \phi, \mathrm{w})=\left\{\begin{array}{l}
\mathrm{T} \text { if } \mathrm{V}_{\mathrm{M}}(\phi, \mathrm{w})=\mathrm{F}, \\
\mathrm{F} \text { otherwise. }
\end{array}\right. \\
& (\wedge) \mathrm{V}_{\mathrm{M}}(\phi \wedge \psi, \mathrm{w})=\left\{\begin{array}{l}
\mathrm{T} \text { if } \mathrm{V}_{\mathrm{M}}(\phi, \mathrm{w})=\mathrm{V}_{\mathrm{M}}(\psi, \mathrm{w})=\mathrm{T}, \\
\mathrm{F} \text { otherwise. }
\end{array}\right. \\
& \left(\square_{\mathrm{K}}\right) \mathrm{V}_{\mathrm{M}}(\square \phi, \mathrm{w})=\left\{\begin{array}{l}
\mathrm{T} \text { if for every } \mathrm{w}^{\prime} \in \mathrm{W}_{\mathrm{M}} \text { such that } \mathrm{wR}_{\mathrm{M}} \mathrm{w}^{\prime}, \mathrm{V}_{\mathrm{M}}\left(\phi, \mathrm{w}^{\prime}\right)=\mathrm{T}, \\
\mathrm{F} \text { otherwise } .
\end{array}\right. \\
& \left(\diamond_{\mathrm{K}}\right) \mathrm{V}_{\mathrm{M}}(\diamond \phi, \mathrm{w})=\left\{\begin{array}{l}
\mathrm{F} \text { if for every } \mathrm{w}^{\prime} \in \mathrm{W}_{\mathrm{M}} \text { such that } \mathrm{wR}_{\mathrm{M}} \mathrm{w}^{\prime}, \mathrm{V}_{\mathrm{M}}\left(\phi, \mathrm{w}^{\prime}\right)=\mathrm{F}, \\
\mathrm{T} \text { otherwise. }
\end{array}\right.
\end{aligned}
$$

I am going to refer to this familiar account of the semantics of modal propositional logic as K-semantics.

Clearly, K-semantics provides a solution to the actualist task of specifying which sets of valuations are admissible. Let us say that the set of MPL-valuations generated by a $\mathrm{K}$-model $\mathrm{M}$ is the following set:

$$
\left\{\mathrm{v} \text { : for some } \mathrm{w} \text { in } \mathrm{W}_{\mathrm{M}} \text {, for every } \mathrm{MPL}-\text { sentence } \phi, \mathrm{V}_{\mathrm{M}}(\phi, \mathrm{w})=\mathrm{v}(\phi)\right\}
$$

We can now formulate in the following terms the solution to the actualist task provided by K-semantics: a set of MPL-valuations is admissible just in case it is generated by some K-model.

However, I am going to argue that this solution is not essentially actualist. Of course, the formal tools deployed in K-semantics are strongly reminiscent of the possibilist

\footnotetext{
$\overline{{ }^{7} \text { K-models are sometimes known }}$ as standard models.
} 
metaphysical picture. Possible worlds bearing relations to one another and bestowing truth values on propositions is precisely how the possibilist conceives of modal reality. But the metaphysical interpretation of the formal tools is not mandatory and is certainly not part of the Kripkean proposal. The actualist is perfectly free to use the formalism without incurring the metaphysical commitments that it might suggest.

What prevents K-semantics from being essentially actualist is not the language employed, but the fact that it has the resources for accommodating possibilist disagreements. K-semantics will be able to represent possibilist disagreements insofar as different K-models can generate the same set of MPL-valuations. The possibilist will be able to take these models to represent different accounts of modal reality that agree on which valuations it contains. I am going to present in the remainder of this section specific ways in which K-semantics can accommodate possibilist disagreements. It will emerge that a set of MPL-valuations will never be generated by a unique K-model - if a set of MPL-valuations is generated by any K-model, it will be generated by many Kmodels. I want to consider three obstacles to unique generation.

I. I want to consider first an obstacle to uniqueness that is trivial and innocuous. If a set of MPL-valuations V is generated by a K-model M, it will also be generated by a K-model in which $\mathrm{W}_{\mathrm{M}}$ is replaced with any other set $\mathrm{S}$ of the same cardinality. If $\mathrm{h}$ is a one-to-one correspondence from $\mathrm{W}_{\mathrm{M}}$ to $\mathrm{S}$, all we need to do to get another model that also generates $\mathrm{V}$ is to replace $\mathrm{R}_{\mathrm{M}}$ with the relation pairing the images under $h$ of the elements of $\mathrm{W}_{M}$ paired by $\mathrm{R}_{\mathrm{M}}$, and $\mathrm{S}_{\mathrm{M}}$ with the function pairing $\alpha$ and $\mathrm{h}(\mathrm{w})$ with $\mathrm{S}_{\mathrm{M}}(\alpha, \mathrm{w})$, for every atomic sentence $\alpha$ and every $\mathrm{w}$ in $\mathrm{W}_{\mathrm{M}}$. What renders this obstacle to uniqueness trivial is the obvious isomorphism between $\mathrm{M}$ and the resulting $\mathrm{K}$-model, as the highest level of uniqueness to which we can aspire with formal objects is uniqueness up to isomorphism. If $\mathrm{V}$ is generated by multiple K-models, but these are isomorphic to one another, then, for all intents and purposes, $\mathrm{V}$ is uniquely generated.

II. The second obstacle to uniqueness that I want to consider involves non-isomorphic models. It arises from the possibility of K-models containing worlds that agree with one another on the truth value of every MPL-sentence. One instance of this phenomenon is discussed by Hughes and Cresswell (1984: 48), who show that for every $\mathrm{K}$-model $\mathrm{M}$, there is another $\mathrm{K}$-model in which every element of $\mathrm{W}_{\mathrm{M}}$ is replaced by two worlds that agree with one another on the truth value that they assign to each MPL-sentence. If $\mathrm{V}$ is generated by $\mathrm{M}$, then it will also be generated by the models that we obtain in this way. And all these models will be nonisomorphic. Any set of MPL-valuations that is generated by a K-model is generated by multiple non-isomorphic K-models.

III. The third obstacle to uniqueness that I want to consider concerns the accessibility relation. Let $\mathrm{M}$ be a $\mathrm{K}$-model with the following features:

a. $\mathrm{W}_{\mathrm{M}}$ contains a world $\mathrm{w}^{*}$ from which denumerably many worlds are accessible, and a world $\mathrm{w}^{+}$not accessible from $\mathrm{w}^{*}$.

b. The same worlds are accessible from $\mathrm{w}^{+}$and from each of the worlds that are accessible from $\mathrm{w}^{*}$.

c. For some enumeration of the atomic sentences, $\alpha_{1}, \alpha_{2}, \alpha_{3} \ldots$, and some enumeration of the worlds that are accessible from $\mathrm{w}^{*}, \mathrm{w}_{1}, \mathrm{w}_{2}, \mathrm{w}_{3} \ldots$, for every positive 
integer $\mathrm{n}$, we have that the first $\mathrm{n}$ atomic sentences, and only those, are true at the $\mathrm{nth}$ world, i.e., for all positive integers $\mathrm{n}, \mathrm{m}, \mathrm{S}_{\mathrm{M}}\left(\alpha_{\mathrm{m}}, \mathrm{w}_{\mathrm{n}}\right)=\mathrm{T}$ iff $\mathrm{m} \leq \mathrm{n}$.

d. Every atomic sentence is true at $\mathrm{w}^{+}$.

Now, compare $\mathrm{M}$ with the $\mathrm{K}$-model $\mathrm{M}^{\prime}$ that we obtain from $\mathrm{M}$ by making $\mathrm{w}^{+}$ accessible from $\mathrm{w}^{*}$, i.e., $\left.\mathrm{M}^{\prime}=<\mathrm{W}_{\mathrm{M}}, \mathrm{R}_{\mathrm{M}} \cup\left\{<\mathrm{w}^{*}, \mathrm{w}^{+}\right\rangle\right\}, \mathrm{S}_{\mathrm{M}}>$. I claim that $\mathrm{M}$ and $\mathrm{M}^{\prime}$ generate the same set of MPL-valuations, since, for every MPL-sentence $\phi$ and every $\mathrm{w} \in \mathrm{W}_{\mathrm{M}}, \mathrm{V}_{\mathrm{M}}(\phi, \mathrm{w})=\mathrm{V}_{\mathrm{M}^{\prime}}(\phi, \mathrm{w})$. Clearly, this claim could fail only if it failed for $\mathrm{w}^{*}$, i.e., if for some MPL-sentence $\psi, \mathrm{V}_{\mathrm{M}}\left(\psi, \mathrm{w}^{*}\right) \neq \mathrm{V}_{\mathrm{M}^{\prime}}\left(\psi, \mathrm{w}^{*}\right)$. This, in turn, could obtain only if there was some MPL-sentence $\gamma$ such that $\mathrm{V}_{\mathrm{M}}\left(\gamma, \mathrm{w}_{\mathrm{i}}\right)=\mathrm{T}$ for every positive integer i but $\mathrm{V}_{\mathrm{M}}\left(\gamma, \mathrm{w}^{+}\right)=\mathrm{F}$ (then we would have $\mathrm{V}_{\mathrm{M}}\left(\square \gamma, \mathrm{w}^{*}\right)=\mathrm{T}, \mathrm{V}_{\mathrm{M}^{\prime}}\left(\square \gamma, \mathrm{w}^{*}\right)=\mathrm{F}$ ) or if $\mathrm{V}_{\mathrm{M}}\left(\gamma, \mathrm{w}_{\mathrm{i}}\right)=\mathrm{F}$ for every positive integer i but $\mathrm{V}_{\mathrm{M}}\left(\gamma, \mathrm{w}^{+}\right)=\mathrm{T}$ (then we would have $\left.\mathrm{V}_{\mathrm{M}}\left(\diamond \gamma, \mathrm{w}^{*}\right)=\mathrm{F}, \mathrm{V}_{\mathrm{M}^{\prime}}\left(\diamond \gamma, \mathrm{w}^{*}\right)=\mathrm{T}\right)$. Hence, to establish our claim, it would suffice to show that if for all $\mathrm{m}, \mathrm{n}, \mathrm{V}_{\mathrm{M}}\left(\phi, \mathrm{w}_{\mathrm{m}}\right)=\mathrm{V}_{\mathrm{M}}\left(\phi, \mathrm{w}_{\mathrm{n}}\right)$, then $\mathrm{V}_{\mathrm{M}}\left(\phi, \mathrm{w}^{+}\right)=\mathrm{V}_{\mathrm{M}}\left(\phi, \mathrm{w}_{\mathrm{n}}\right)$ (for every $\mathrm{n}$ ). This can be easily shown. ${ }^{8}$ We can conclude that the set of MPL-valuations generated by $\mathrm{M}$ is also generated by $\mathrm{M}^{\prime}$, even though these two models are not isomorphic.

We have seen in this section that multiple generation is a widespread phenomenon in $\mathrm{K}$-semantics. There are many cases in which different K-models generate the same set of MPL-valuations. This will enable the possibilist to use K-semantics to accommodate possibilist disagreements. K-semantics is not essentially actualist. It has, so to speak, moving parts that the actualist does not need.

\section{Other Options}

We can achieve unique generation without abandoning K-semantics. All we need to do is define a subset of the set of K-models containing, for every set of MPL-valuations that is generated by some K-model, exactly one K-model that generates it. We can achieve this with Kit Fine's notion of natural models (Fine 1975: 23). Natural models are K-models that are differentiated and tight. A K-model $\mathrm{M}$ is differentiated just in case it satisfies the following condition.

For all $\mathrm{w}, \mathrm{w}^{\prime} \in \mathrm{W}_{\mathrm{M}}$, if $\mathrm{w} \neq \mathrm{w}^{\prime}$, then for some MPL-sentence $\phi, \mathrm{V}_{\mathrm{M}}(\phi$, w) $\neq \mathrm{V}_{\mathrm{M}}\left(\phi, \mathrm{w}^{\prime}\right)$.

A K-model $\mathrm{M}$ is tight just in case it satisfies this condition:

For all $w, w^{\prime} \in \mathrm{W}_{\mathrm{M}}, \mathrm{wR}_{\mathrm{M}} \mathrm{w}^{\prime}$ if for every MPL-sentence $\phi$,

(1) if $\mathrm{V}_{\mathrm{M}}(\square \phi, \mathrm{w})=\mathrm{T}$, then $\mathrm{V}_{\mathrm{M}}\left(\phi, \mathrm{w}^{\prime}\right)=\mathrm{T}$, and

(2) if $\mathrm{V}_{\mathrm{M}}(\diamond \phi, \mathrm{w})=\mathrm{F}$, then $\mathrm{V}_{\mathrm{M}}\left(\phi, \mathrm{w}^{\prime}\right)=\mathrm{F}$.

Focusing on natural models does nothing about the first, innocuous obstacle to unique generation (I), but the two substantial obstacles are removed. Differentiated

\footnotetext{
${ }^{8}$ We can show with a simple induction that for every MPL-sentence $\phi$, there is a positive integer $\mathrm{n}$ such that beyond $\mathrm{w}_{\mathrm{n}}$, the truth value of $\phi$ does not change, i.e., for every positive integer $m \geq n, \mathrm{~V}_{\mathrm{M}}\left(\phi, \mathrm{w}_{\mathrm{m}}\right)=\mathrm{V}_{\mathrm{M}}(\phi$, $\mathrm{w}_{\mathrm{n}}$ ). Call this the limit value of $\phi$. We can then show, again by induction, that the value of $\phi$ at $\mathrm{w}^{+}$is its limit value. It is a straightforward corollary of this that if for all $\mathrm{m}, \mathrm{n}, \mathrm{V}_{\mathrm{M}}\left(\phi, \mathrm{w}_{\mathrm{m}}\right)=\mathrm{V}_{\mathrm{M}}\left(\phi, \mathrm{w}_{\mathrm{n}}\right)$, then $\mathrm{V}_{\mathrm{M}}(\phi$, $\left.\mathrm{w}^{+}\right)=\mathrm{V}_{\mathrm{M}}\left(\phi, \mathrm{w}_{\mathrm{n}}\right)$ (for every $\left.\mathrm{n}\right)$.
} 
models do not have worlds that agree with each other on the truth values of all sentences (II), and tight models that disagree on the extension of the accessibility relation will generate different sets of valuations (III). Up to isomorphism, every set of MPL-valuations that is generated by some K-model is generated by a unique natural Kmodel.

I want to argue, however, that achieving unique generation in this way does not amount to providing an essentially actualist semantics. To be an essentially actualist semantics, a semantics must single out the admissible sets of MPL-valuations without drawing on formal resources that would enable us to make room for possibilist disagreements. Focusing on natural models does not satisfy this requirement. We have defined the set of natural models as a subset of the set of K-models, and the definition of the set of K-models does draw on resources that suffice for making sense of possibilist disagreements. This strategy invokes these resources to define K-models and then cancels them out by concentrating on natural models. An essentially actualist semantics should not employ these resources to start with. It should single out the admissible sets of MPL-valuations without drawing at any stage on resources that would suffice for making sense of possibilist disagreements.

To find an essentially actualist semantics, we need to abandon the formal tools of Ksemantics, possible worlds and the accessibility relation, and attempt to specify without their help which sets of MPL-valuations are admissible. We start our search by going back to the semantics for modal propositional logic put forward by Rudolf Carnap (1956: 182). On Carnap's approach, there is exactly one admissible set of MPLvaluations. It is given by a binary function $\mathrm{C}$ pairing each MPL-sentence and atomic valuation with a truth value. $\mathrm{C}$ is defined by recursion. For every atom $\alpha$ and every atomic valuation $\sigma$, we have that $\mathrm{C}(\alpha, \sigma)=\sigma(\alpha)$. The recursive clauses for the modal operators take the following form, for every MPL-sentence $\phi$, and every atomic valuation $\sigma$ :

$$
\begin{aligned}
& \left(\square_{\mathrm{C}}\right) \mathrm{C}(\square \phi, \sigma)=\left\{\begin{array}{l}
\mathrm{T} \text { if for every atomic valuation } \sigma^{\prime}, \mathrm{C}\left(\phi, \sigma^{\prime}\right)=\mathrm{T}, \\
\mathrm{F} \text { otherwise. }
\end{array}\right. \\
& \left(\diamond_{\mathrm{C}}\right) \mathrm{C}(\diamond \phi, \sigma)=\left\{\begin{array}{l}
\mathrm{F} \text { if for every atomic valuation } \sigma^{\prime}, \mathrm{C}\left(\phi, \sigma^{\prime}\right)=\mathrm{F}, \\
\mathrm{T} \text { otherwise. }
\end{array}\right.
\end{aligned}
$$

The set of extensions to MPL of the atomic valuations that we obtain in this way is, on Carnap's approach, the one and only admissible set of MPL-valuations.

Carnap's approach is overly restrictive in several ways. One obvious problem is that it does not treat as admissible any sets of MPL-valuations that do not contain extensions of every atomic valuation. This is fine if we want the modal operators to express logical necessity and possibility, but it is overly restrictive if we want our modal operators to express metaphysical modalities. Treated as an account of metaphysical necessity and possibility, Carnap's system carries a commitment to the view that everything that is logically possible is metaphysically possible, and only what is logically necessary is metaphysically necessary.

The commitment is easily discharged with a minor modification of the Carnapian framework, presented in Kripke's paper, "A Completeness Theorem in Modal Logic" 
(Kripke 1959). Once we accept that some atomic valuations might be impossible, we can no longer expect a unique MPL-extension of a possible atomic valuation $\sigma$ that agrees with the meanings of the truth-functional connectives and modal operators. Which MPL-extension of $\sigma$ satisfies this requirement will depend on which other atomic valuations are contained in modal reality. Hence, instead of defining a single function pairing each MPL-sentence and atomic valuation with a truth value, we need to define a function of this kind for every hypothesis as to which atomic valuations are contained in modal reality - for every set of atomic valuations.

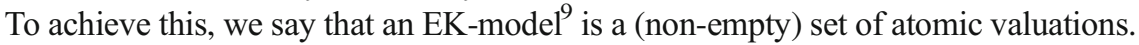
For every EK-model $\Sigma$, we define a function $\mathrm{V}_{\Sigma}$, the canonical extension of $\Sigma$, pairing each MPL-sentence and element of $\Sigma$ with a unique truth value. The definition then proceeds along the same lines as the definition of $\mathrm{C}$, above, with the set of all atomic valuations replaced by $\Sigma$. Hence, the clauses for the modal operators now read as follows, for every MPL-sentence $\phi$, and every $\sigma \in \Sigma$ :

$$
\begin{aligned}
& \left(\square_{\mathrm{EK}}\right) \mathrm{V}_{\Sigma}(\square \phi, \sigma)=\left\{\begin{array}{l}
\mathrm{T} \text { if for every } \sigma^{\prime} \in \Sigma, \mathrm{V}_{\Sigma}\left(\phi, \sigma^{\prime}\right)=\mathrm{T}, \\
\mathrm{F} \text { otherwise. }
\end{array}\right. \\
& \left(\diamond_{\mathrm{EK}}\right) \mathrm{V}_{\Sigma}(\diamond \phi, \sigma)=\left\{\begin{array}{l}
\mathrm{F} \text { if for every } \sigma^{\prime} \in \Sigma, \mathrm{V}_{\Sigma}\left(\phi, \sigma^{\prime}\right)=\mathrm{F}, \\
\mathrm{T} \text { otherwise. }
\end{array}\right.
\end{aligned}
$$

We can now define, for every EK-model $\Sigma$, the set of MPL-valuations generated by $\Sigma$ as the following set:

$$
\left\{\mathrm{v} \text { : for some } \sigma \text { in } \Sigma \text {, for every MPL-sentence } \phi, \mathrm{V}_{\Sigma}(\phi, \sigma)=\mathrm{v}(\phi)\right\}
$$

This gives us a solution to the actualist task: a set of MPL-valuations is admissible just in case it is generated by some EK-model. Furthermore, this solution is essentially actualist. For any two different EK-models, there is at least one atomic valuation $\sigma$ that is a member of one but not of the other. Hence, the set of MPL-valuations generated by one of the EK-models, but not the set generated by the other, will contain an extension of $\sigma$. Therefore, different EK-models will generate different sets of MPL-valuations, as required for essentially actualist status. Furthermore, the definition has not invoked at any stage formal tools that would enable us to make sense of possibilist disagreements.

Nevertheless, the actualist would be ill advised to adopt EK-semantics as her account of which sets of MPL-valuations are admissible, as it rules out features of sets of MPL-valuations that the actualist might well regard as compatible with the meanings of the modal operators. The questionable restrictions include the following:

1. A set of MPL-valuations will be admissible only if all the valuations in the set agree on the truth value of each modal sentence.

2. Two different admissible sets of MPL-valuations cannot extend the same set of atomic valuations.

\footnotetext{
9 'EK' abbreviates 'early Kripke'.
} 
Neither of these restrictions is mandated by the actualist position. Contrary to 1 , an actualist may want to regard an account of modal reality as coherent even though it represents it as containing valuations that disagree on the truth values of some modal sentences. Against 2, an actualist may want to make room for the possibility of coherent accounts of modal reality that agree on which atomic valuations it contains but disagree on how these are extended to modal sentences. Although EK-semantics is essentially actualist, an actualist who does not accept these restrictions will not be able to use it to specify which accounts of modal reality are compatible with the meanings of the modal operators.

The actualist might try to remove some of these restrictions without returning to $\mathrm{K}$-semantics by introducing an accessibility relation directly on atomic valuations. To achieve this, we say that an A-model ${ }^{10} \mathrm{M}$ is an ordered pair $\left\langle\Sigma_{M}, R_{M}>\right.$, where $\Sigma_{M}$ is a (non-empty) set of atomic valuations and $R_{M}$ is a binary relation on $\Sigma_{M}$. For every A-model $\mathrm{M}$, we define a function $\mathrm{V}_{\mathrm{M}}$, the canonical extension of $\Sigma_{\mathrm{M}}$ (with respect to $\mathrm{M}$ ), pairing each MPL-sentence and element of $\Sigma_{\mathrm{M}}$ with a unique truth value. The definition proceeds along the same lines as for K-semantics. ${ }^{11}$ The clauses for the modal operators now go as follows, for every MPL-sentence $\phi$ and every $\sigma \in \Sigma_{\mathrm{M}}$ :

$$
\begin{aligned}
& \left(\square_{\mathrm{A}}\right) \mathrm{V}_{\mathrm{M}}(\square \phi, \sigma)=\left\{\begin{array}{l}
\mathrm{T} \text { if for every } \sigma^{\prime} \in \Sigma_{\mathrm{M}} \text { such that } \sigma \mathrm{R}_{\mathrm{M}} \sigma^{\prime}, \mathrm{V}_{\mathrm{M}}\left(\phi, \sigma^{\prime}\right)=\mathrm{T}, \\
\mathrm{F} \text { otherwise. }
\end{array}\right. \\
& \left(\diamond_{\mathrm{A}}\right) \mathrm{V}_{\mathrm{M}}(\diamond \phi, \sigma)=\left\{\begin{array}{l}
\mathrm{F} \text { if for every } \sigma^{\prime} \in \Sigma_{\mathrm{M}} \text { such that } \sigma \mathrm{R}_{\mathrm{M}} \sigma^{\prime}, \mathrm{V}_{\mathrm{M}}\left(\phi, \sigma^{\prime}\right)=\mathrm{F}, \\
\mathrm{T} \text { otherwise. }
\end{array}\right.
\end{aligned}
$$

We define as before the function pairing each A-model with the set of MPLvaluations it generates, and we say that the admissible sets are those that are generated by some A-model.

This move succeeds in removing the restrictions on admissible sets of MPLvaluations that we mentioned above (1 and 2). By introducing $\mathrm{R}_{\mathrm{M}}$, we are able to count some sets of MPL-valuations as admissible even though they contain valuations that disagree on the values of modal sentences (1). We also make room for different admissible sets of MPL-valuations that extend the same set of atomic valuations (2).

Nevertheless, A-semantics still imposes restrictions on admissible sets of MPL-valuations that an actualist might not want to endorse. As we have just seen, an A-model can generate a set of MPL-valuations containing valuations that disagree with one another on the values they assign to some modal sentences. However, an A-model cannot generate a set of MPL-valuations containing valuations that disagree with one another on the values they assign to some modal sentences while agreeing on the value that they assign to each nonmodal sentence. A-semantics imposes the following restriction on admissible sets of MPL-valuations:

\footnotetext{
10 'A' abbreviates 'accessibility'.

${ }^{11}$ This approach can be found in the work of Kanger (1957: 33).
} 
3. An admissible set of MPL-valuations cannot contain different valuations that extend the same atomic valuation.

Notice that 3 is weaker than 1. Every set of MPL-valuations that satisfies 1 also satisfies 3 , but a set might satisfy 3 and violate 1 . Nevertheless, the actualist might still not recognize this restriction as a consequence of her position. She might see nothing wrong with modal reality containing valuations that agree with one another concerning the values of all non-modal sentences but disagree about the value of some modal sentences. But Asemantics lacks the resources to represent modal reality as having this feature.

With respect to unique generation, the results are mixed. In the absence of worlds, the first two obstacles to uniqueness (I and II) disappear. The third (III), however, remains. We can easily replicate in A-models the situation we described in section 3 of two models that generate the same set of MPL-valuations but disagree on the extension of the accessibility relation. A-semantics is not essentially actualist. ${ }^{12}$

We still have not found an essentially actualist semantics that does not impose restrictions on which sets of MPL-valuations are admissible that do not follow from the actualist position. My goal in the remainder is to describe a semantics for MPL that satisfies this description - an essentially actualist semantics that agrees with Ksemantics on which sets of MPL-valuations are admissible.

\section{EA-Semantics}

A semantics for MPL needs to specify which sets of MPL-valuations are admissible. Ksemantics achieves this in a rather roundabout way. It says that a set of MPL-valuations is admissible just in case it is generated by some K-model.

The alternative semantic framework that I am going to introduce will take a much more direct approach to the task. We will dispense with possible worlds and the accessibility relation, in terms of which K-models are defined. Instead, we will specify directly the conditions that a set of MPL-valuations needs to satisfy in order to be admissible.

Let us say that an MPL-valuation $\mathrm{v}$ is Boolean just in case it agrees with the meanings of the truth-functional connectives, i.e., just in case it satisfies the following conditions, for all MPL-sentences $\phi, \psi$ :

$$
\begin{gathered}
\mathrm{v}(\phi) \neq \mathrm{v}(\neg \phi) \\
\mathrm{v}(\phi \wedge \psi)=\operatorname{Tiff} \mathrm{v}(\phi)=\mathrm{v}(\psi)=\mathrm{T}
\end{gathered}
$$

A necessary condition for a set of MPL-valuations to be admissible is that all the valuations in the set are Boolean. This will ensure that the set is compatible with the meanings of the truth-functional connectives. All we need to do now is to specify

\footnotetext{
12 On the assumption, that is, that we want our semantics to be in principle compatible with all normal modal systems. If we maintained, e.g., that it follows from the meanings of the modal operators that accessibility has to be the total relation on the set of atomic valuations contained in modal reality, this obstacle to essential actualism would be neutralized. I am indebted on this point to an anonymous referee for this journal.
} 
conditions that will ensure that a set of Boolean MPL-valuations agrees with the meanings of the modal operators.

We can motivate the approach I am going to adopt by reflecting on how the truth values that MPL-sentences receive from the worlds in a K-model restrict the extension of the accessibility relation. $\mathrm{w}_{2}$ cannot be accessible from $\mathrm{w}_{1}$ unless all the necessities of $\mathrm{w}_{1}$ are true in $\mathrm{w}_{2}$ and all the impossibilities of $\mathrm{w}_{1}$ are false in $\mathrm{w}_{2}$. This is a necessary condition for $\mathrm{w}_{2}$ being accessible from $\mathrm{w}_{1}$. But it is not a sufficient condition. $\mathrm{w}_{2}$ might fail to be accessible from $\mathrm{w}_{1}$ even if the condition is satisfied. This is, in effect, the source of the third obstacle (III) to unique generation that we considered in section 3 . The obstacle would disappear if we turned this into a sufficient as well as necessary condition for accessibility. ${ }^{13}$ What I propose to do is to abandon accessibility and formulate the semantics for MPL in terms of this condition.

Let us say that an MPL-valuation v' actualizes an MPL-valuation v just in case every necessity of $v$ is true in $v^{\prime}$ and every impossibility of $v$ is false in $v^{\prime}$, i.e.,:

$$
\begin{aligned}
& \mathrm{v}^{\prime} \text { actualizes } \mathrm{v} \text { just in case for every MPL-sentence } \phi, \\
& \text { if } \mathrm{v}(\square \phi)=\mathrm{T} \text {, then } \mathrm{v}(\phi)=\mathrm{T} \text {, and if } \mathrm{v}(\diamond \phi)=\mathrm{F} \text {, then } \mathrm{v}^{\prime}(\phi)=\mathrm{F} \text {. }
\end{aligned}
$$

We can use the notion of actualization as the basis for our modal semantics. We say that an EA-model ${ }^{14}$ is a set $\mathrm{V}$ of Boolean valuations satisfying the following conditions, for every MPL-sentence $\phi$ and every $\mathrm{v} \in \mathrm{V}$ :

$$
\begin{aligned}
& \left(\square_{\mathrm{EA}}\right) \mathrm{v}(\square \phi)=\mathrm{T} \text { if for every } \mathrm{v}^{\prime} \in \mathrm{V}, \text { such that } \mathrm{v}^{\prime} \text { actualizes } \mathrm{v}, \mathrm{v}^{\prime}(\phi)=\mathrm{T} . \\
& \left(\diamond_{\mathrm{EA}}\right) \mathrm{v}(\diamond \phi)=\mathrm{F} \text { if for every } \mathrm{v}^{\prime} \in \mathrm{V}, \text { such that } \mathrm{v}^{\prime} \text { actualizes } \mathrm{v}, \mathrm{v}^{\prime}(\phi)=\mathrm{F} \text {. }
\end{aligned}
$$

Notice that we do not need to require, in addition, that $\square \phi$ is false in $v$ if $\phi$ is false in some valuation that actualizes $\mathrm{v}$. If $\square \phi$ is true in $\mathrm{v}$ and $\phi$ is false in $\mathrm{v}^{\prime}$, then $\mathrm{v}^{\prime}$ does not actualize $v$. Likewise, we do not need to require that $\nabla \phi$ is true in $v$ if $\phi$ is true in some valuation that actualizes $\mathrm{v}$. If $\nabla \phi$ is false in $\mathrm{v}$ and $\phi$ is true in $\mathrm{v}^{\prime}$, then $\mathrm{v}^{\prime}$ does not actualize $v$. This means that a set of Boolean valuations can fail to be an EA-model only if it contains a valuation that either fails to treat as necessary what is true in every valuation that actualizes it or fails to treat as impossible what is false in every valuation that actualizes it.

I would like to emphasize that an EA-model does not consist in a set of MPLvaluations and a binary relation (actualization) that satisfy certain conditions. We have not simply replaced accessibility with actualization in the definition of A-models. An EA-model consists in a set of MPL-valuations that satisfies certain conditions. We have introduced the notion of actualization in order to provide a perspicuous formulation of the conditions that a set of MPL-valuations needs to satisfy in order to be an EA-model. But the notion is entirely dispensable. $\left(\square_{\mathrm{EA}}\right)$ and $\left(\vee_{\mathrm{EA}}\right)$ can be easily formulated without

\footnotetext{
${ }_{13}$ See our discussion above of Fine's tight models.

14 'EA' abbreviates 'essentially actualist'.
} 
its help ( $\phi$ and $\psi$ range over MPL-sentences and $v$ and $v^{\prime}$ over elements of $V$ ):

$$
\begin{gathered}
(\square \mathrm{EA})(\forall \phi)(\forall \mathrm{v})\left(\left(\forall \mathrm{v}^{\prime}\right)\left[(\forall \psi)\left(\left(\mathrm{v}(\square \psi)=\mathrm{T} \rightarrow \mathrm{v}^{\prime}(\psi)=\mathrm{T}\right) \wedge\left(\mathrm{v}(\diamond \psi)=\mathrm{F} \rightarrow \mathrm{v}^{\prime}(\psi)=\mathrm{F}\right)\right) \rightarrow \mathrm{v}^{\prime}(\phi)=\mathrm{T}\right] \rightarrow \mathrm{v}(\square \phi)=\mathrm{T}\right) \\
\left(\diamond_{\mathrm{EA}}\right)(\forall \phi)(\forall \mathrm{v})\left(\left(\forall \mathrm{v}^{\prime}\right)\left[(\forall \psi)\left(\left(\mathrm{v}(\square \psi)=\mathrm{T} \rightarrow \mathrm{v}^{\prime}(\psi)=\mathrm{T}\right) \wedge\left(\mathrm{v}(\diamond \psi)=\mathrm{F} \rightarrow \mathrm{v}^{\prime}(\psi)=\mathrm{F}\right)\right) \rightarrow \mathrm{v}^{\prime}(\phi)=\mathrm{F}\right] \rightarrow \mathrm{v}(\diamond \phi)=\mathrm{F}\right)
\end{gathered}
$$

$\left(\square_{\mathrm{EA}}\right)$ and $\left(\diamond_{\mathrm{EA}}\right)$ are exclusively concerned with the values that the members of $\mathrm{V}$ assign to each MPL-sentence.

We can now provide a perfectly straightforward solution to the actualist semantic task: a set of MPL-valuations is admissible if and only if it is an EA-model. This gives us what we were looking for-a specification of which sets of MPL-valuations are admissible that is essentially actualist but does not impose any restrictions on admissible sets that the actualist might not want to adopt.

The claim that EA-semantics is essentially actualist is self-evident. An EA-model is a set of MPL-valuations. Hence, by the extensionality of sets, different EA-models cannot correspond to the same set of MPL-valuations. EA-semantics lacks the resources for representing possibilist disagreements.

We can also easily show that EA-semantics does not impose any restrictions on which sets of MPL-valuations are admissible that the actualist might not want to endorse. We can show, in fact that, EA-semantics imposes exactly the same restrictions as K-semantics. This is an immediate consequence of the following result:

A set of MPL-valuations is an EA-model if and only if it is generated by some Kmodel. $^{15}$

Clearly, it follows from this that none of the restrictions on admissible sets of MPLvaluations that we invoked to motivate the rejection of other semantic frameworks are imposed by EA-semantics, but it will be interesting to look at some EA-models that violate them.

For a violation of restriction 3, and a fortiori of restriction 1 , let $\sigma$ be an atomic valuation, let $\mathrm{v}$ be the Boolean extension of $\sigma$ to MPL such that for every MPLsentence $\phi, \mathrm{v}(\square \phi)=\mathrm{T}, \mathrm{v}(\diamond \phi)=\mathrm{F}$, and let $\mathrm{v}^{\prime}$ be the Boolean extension of $\sigma$ to MPL such that for every MPL-sentence $\phi, v^{\prime}(\square \phi)=v^{\prime}(\diamond \phi)=v(\phi)$. We can easily show that $\left\{\mathrm{v}, \mathrm{v}^{\prime}\right\}$ is an EA-model in which $\mathrm{v}$ actualizes $\mathrm{v}^{\prime}$ but not itself and $\mathrm{v}^{\prime}$ does not actualize itself or $\mathrm{v}$. And $\left\{\mathrm{v}, \mathrm{v}^{\prime}\right\}$ violates restriction 3. For $\mathrm{v}$ and $\mathrm{v}^{\prime}$ are MPL-valuations that agree with

\footnotetext{
${ }^{15}$ For the right-to-left direction, let V be a set of MPL-valuations that's generated by some K-model. We need to show that $\mathrm{V}$ is a set of Boolean valuations satisfying $\square_{\mathrm{EA}}$ and $\diamond_{\mathrm{EA}}$.

Let $\mathrm{M}$ be a $\mathrm{K}$-model that generates $\mathrm{V}$. Let $\mathrm{v} \in \mathrm{V}$. Then, there is a $\mathrm{w} \in \mathrm{W}_{\mathrm{M}}$ such that for every MPLsentence $\phi, \mathrm{V}_{\mathrm{M}}(\phi, \mathrm{w})=\mathrm{v}(\phi)$. Let $\mathrm{w}$ be an element of $\mathrm{W}_{\mathrm{M}}$ satisfying this condition.

We show first that $v$ is Boolean. By the definition of $\mathrm{V}_{\mathrm{M}}$ we have that for every MPL-sentence $\phi, \mathrm{V}_{\mathrm{M}}(\phi$, $\mathrm{w}) \neq \mathrm{V}_{\mathrm{M}}(\neg \phi, \mathrm{w})$ and for all MPL-sentences $\phi, \psi, \mathrm{V}_{\mathrm{M}}(\phi \wedge \psi, \mathrm{w})=\mathrm{T}$ iff $\mathrm{V}_{\mathrm{M}}(\phi, \mathrm{w})=\mathrm{V}_{\mathrm{M}}(\psi, \mathrm{w})=\mathrm{T}$. It follows that, for every MPL-sentence $\phi, \mathrm{v}(\phi) \neq \mathrm{v}(\neg \phi)$ and for all MPL-sentences $\phi, \psi, \mathrm{v}(\phi \wedge \psi)=\mathrm{T}$ iff $\mathrm{v}(\phi)=\mathrm{v}(\psi)=\mathrm{T}$.

For $\square_{\mathrm{EA}}$, assume that for every $\mathrm{v}^{\prime} \in \mathrm{V}$ that actualizes $\mathrm{v}, \mathrm{v}^{\prime}(\phi)=\mathrm{T}$. If $\mathrm{wR}_{\mathrm{M}^{\prime}} \mathrm{w}^{\prime}$ and we let $\mathrm{v}^{\prime}(\psi)=\mathrm{V}_{\mathrm{M}}\left(\psi, \mathrm{w}^{\prime}\right)$ for every MPL-sentence $\psi$, then $v^{\prime}$ actualizes $v$. Hence, for every $w^{\prime} \in \mathrm{W}_{M}$ such that $\mathrm{wR}_{\mathrm{M}^{\prime}} \mathrm{w}^{\prime}, \mathrm{V}_{\mathrm{M}}\left(\phi, \mathrm{w}^{\prime}\right)=\mathrm{T}$. And now it follows by $\square_{\mathrm{K}}$ that $\mathrm{V}_{\mathrm{M}}(\square \phi, \mathrm{w})=\mathrm{T}$.

We deal with $\diamond_{\mathrm{EA}}$ in the same way.

For the left-to-right direction, notice that if $\mathrm{V}$ is an EA-model, then we obtain a K-model $\mathrm{M}$ if we take $\mathrm{V}$ as $\mathrm{W}_{\mathrm{M}}$, the actualization relation on $\mathrm{V}$ as $\mathrm{R}_{\mathrm{M}}$, and the function pairing each element of $\mathrm{V}$ with its restriction to the set of atomic sentences as $\mathrm{S}_{\mathrm{M}}$.
} 
each other on the truth values that they ascribe to the atoms but disagree on the truth values that they ascribe to some modal sentences.

For a violation of restriction 2, let $\sigma$ and $\sigma$ ' be different atomic valuations. Let $\mathrm{v}_{1}$ be the Boolean extension of $\sigma$ to MPL such that for every MPL-sentence $\phi$, $\mathrm{v}_{1}(\square \phi)=\mathrm{v}_{1}(\nabla \phi)=\mathrm{v}_{1}(\phi)$, and let $\mathrm{v}_{1}{ }^{\prime}$ be the Boolean extension of $\sigma^{\prime}$ to MPL such that for every MPL-sentence $\phi, \mathrm{v}_{1}{ }^{\prime}(\square \phi)=\mathrm{T}$ iff $\mathrm{v}_{1}(\phi)=\mathrm{v}_{1}{ }^{\prime}(\phi)=\mathrm{T}$, and $\mathrm{v}_{1}{ }^{\prime}(\diamond \phi)=\mathrm{F}$ iff $\mathrm{v}_{1}(\phi)=\mathrm{v}_{1}{ }^{\prime}(\phi)=\mathrm{F}$. We can easily show that $\left\{\mathrm{v}_{1}, \mathrm{v}_{1}{ }^{\prime}\right\}$ is an EA-model in which $\mathrm{v}_{1}$ actualizes $\mathrm{v}_{1}{ }^{\prime}$ and itself and $\mathrm{v}_{1}{ }^{\prime}$ actualizes itself but not $\mathrm{v}_{1}$.

Now, let $v_{2}$ and $v_{2}{ }^{\prime}$ be the Boolean extensions of $\sigma$ and $\sigma^{\prime}$ to MPL that reverse the treatments of the modal operators. Thus $v_{2}{ }^{\prime}$ is the Boolean extension of $\sigma^{\prime}$ such that for every MPL-sentence $\phi, \mathrm{v}_{2}{ }^{\prime}(\square \phi)=\mathrm{v}_{2}^{\prime}(\diamond \phi)=\mathrm{v}_{2}^{\prime}(\phi)$, and $\mathrm{v}_{2}$ is the Boolean extension of $\sigma$ such that for every MPL-sentence $\phi, \mathrm{v}_{2}(\square \phi)=\mathrm{T}$ iff $\mathrm{v}_{2}(\phi)=\mathrm{v}_{2}{ }^{\prime}(\phi)=\mathrm{T}$, and $\mathrm{v}_{2}(\diamond \phi)=\mathrm{F}$ iff $\mathrm{v}_{2}(\phi)=\mathrm{v}_{2}{ }^{\prime}(\phi)=\mathrm{F}$. $\left\{\mathrm{v}_{2}, \mathrm{v}_{2}{ }^{\prime}\right\}$ is an EA-model in which $\mathrm{v}_{2}{ }^{\prime}$ actualizes $\mathrm{v}_{2}$ and itself and $\mathrm{v}_{2}$ actualizes itself but not $\mathrm{v}_{2}{ }^{\prime} .\left\{\mathrm{v}_{1}, \mathrm{v}_{1}{ }^{\prime}\right\}$ and $\left\{\mathrm{v}_{2}, \mathrm{v}_{2}{ }^{\prime}\right\}$ violate restriction 2 , as they are different admissible sets of MPL-valuations that extend the same set of atomic valuations.

\section{K-Semantics and EA-Semantics}

The semantic properties and relations that we can define in terms of K-models correspond to the modal system $\mathrm{K}$, the weakest normal system. Since EA-models provide an equivalent definition of admissibility, the resulting logical properties and relations also correspond to $\mathrm{K}$.

Using K-models, we can go on to define stronger logical systems by imposing conditions on the accessibility relation. Thus, for example, the system $\mathrm{T}$ results when we focus on K-models in which accessibility is reflexive. Let us say that a K-model M is reflexive just in case $R_{M}$ is a reflexive relation, and let us say that a set of MPLvaluations is admissible just in case it is generated by some reflexive K-model. The logical properties and relations that we obtain from this definition of admissibility correspond to the system $\mathrm{T}$. The same maneuver, with other restrictions on accessibility, generates the semantic frameworks for other extensions of $\mathrm{K}$.

It might seem that by dispensing with the accessibility relation, EA-semantics loses the ability to provide the semantics for modal systems beyond $\mathrm{K}$. However, this is not so, since we can achieve the same effect with restrictions on EA-models. Thus, let us say that an EA-model $\mathrm{V}$ is reflexive just in case every element of $\mathrm{V}$ actualizes itself. We can now say that a set of MPL-valuations is admissible just in case it is a reflexive EAmodel. Using this notion of accessibility, we can define, once more, the logical properties and relations of system $\mathrm{T}$. The same strategy will generate the semantics of other extensions of $\mathrm{K}^{16}$

However, it is by no means clear that EA-models can generate a modal semantics that replicates the success of K-models in the development of model theory. The proofs of many results in the model theory of modal propositional logic make use of features

\footnotetext{
${ }^{16}$ The notion of a frame can also be easily recovered in the new context. Let us say that two EA-models $\mathrm{V}_{1}$, $\mathrm{V}_{2}$ are frame-isomorphic if there is a one-to-one correspondence $\mathrm{h}$ from $\mathrm{V}_{1}$ to $\mathrm{V}_{2}$ such that, for all $\mathrm{v}, \mathrm{v}^{\prime} \in \mathrm{V}_{1}, \mathrm{v}^{\prime}$ actualizes $v$ iff $h\left(v^{\prime}\right)$ actualizes $h(v)$. Then we can define frames as the equivalence classes generated by frameisomorphism.
} 
of K-models that are absent from EA-models, and it remains to be seen whether we can provide alternative proofs based on EA-models. ${ }^{17}$

But my point does not depend on the potential of EA-models as a tool in model theory. My goal has been to show that an essentially actualist semantics for modal propositional logic is possible - that we can specify the admissible sets of MPLvaluations without invoking formal tools that would enable us to make room for possibilist disagreements. The existence of EA-semantics establishes this point, even if we continue to favor K-semantics for the development of model theory.

Acknowledgements I am grateful to Kit Fine, Heejin Kwon, Genoveva Martí, Ben Martin, Chris Peacocke, Stephen Read and Daniel Rothschild for comments on this material, and to Mark Kalderon and Mike Martin for conversations on these issues. I have also benefitted from excellent comments from an anonymous referee for this journal.

Open Access This article is distributed under the terms of the Creative Commons Attribution 4.0 International License (http://creativecommons.org/licenses/by/4.0/), which permits unrestricted use, distribution, and reproduction in any medium, provided you give appropriate credit to the original author(s) and the source, provide a link to the Creative Commons license, and indicate if changes were made.

\section{References}

Adams, R. (1974). Theories of actuality. Nous, 8, 211-231.

Adams, R. (1981). Actualism and thisness. Synthese, 49, 3-41.

Carnap. (1956). Meaning and necessity (2nd ed.). Chicago: University of Chicago Press Original edition, 1947.

Fine, K.. (1975). Some connections between elementary and modal logic. In Proceedings of the third Scandinavian Logic Symposium, edited by S. Kanger. Amsterdam: North-Holland.

Hughes, G. E., \& Cresswell, M. J. (1984). A companion to modal logic. London: Methuen.

Kanger, S. (1957). Provability in logic. Uppsala: Almqvist \& Wiksells.

Kripke, S. (1959). A completeness theorem in modal logic. Journal of Symbolic Logic, 24, 1-14.

Kripke, S. (1963). Semantical analysis of modal logic I: normal modal propositional calculi. Zeitschrift für mathematische Logik und Grundlagen der Mathematik, 9, 67-96.

Plantinga, A. (1976). Actualism and possible worlds. Theoria, 42, 139-160.

\footnotetext{
${ }^{17}$ One example is the standard proof in K-semantics of the result that, say, in K4, if $\square \phi \vee \square \psi$ is a theorem, then either $\square \phi$ or $\square \psi$ is also a theorem, for which there is no obvious correlate in EA-semantics. I owe this point to Kit Fine.
} 